\title{
IMPROVEMENTS TO WALL CORRECTIONS AT THE NASA LANGLEY 14X22-FT SUBSONIC TUNNEL
}

\author{
Venkit Iyer*, David D. Kuhl ${ }^{\dagger}$, Lockheed Martin \\ and \\ Eric L. Walker ${ }^{\ddagger}$, NASA Langley Research Center \\ Hampton, VA
}

\begin{abstract}
The new wall pressure measurement system and the TWICS wall correction system for the $14 \times 22-\mathrm{Ft}$ subsonic tunnel are described. Results from a recent semispan test and a full-span test are presented. Comparison with existing classical methods of correction is shown. A modification of the TWICS code to treat the effect due to a deflected wake from a high-lift wing is also discussed. The current implementation of TWICS for the $14 \times 22-\mathrm{Ft}$ tunnel is shown to be an improvement over existing methods.
\end{abstract}

\section{INTRODUCTION}

Wind tunnel facilities all over the world are constantly seeking improvements in testing capabilities and in the quality of data provided to the customers. The metrics of performance is no longer the number of data points taken, but rather the efficient manner in which the test objectives are achieved by acquiring quality data. Two items of importance in the area of data quality are: statistical analysis of repeatability of data based on periodic check-standard testing ${ }^{1}$, and correction for interference due to the tunnel walls. In particular, wall interference appears as a bias error on the measured data, which needs to be quantified in order to arrive at the equivalent free-air values. It is important to do this accurately because subtle phenomena such as Reynolds number effects can be masked otherwise.

In this drive to improve performance and data quality, wind tunnels are retro-fitted with new hardware, data acquisition systems and analysis software. The $14 \times 22-F t$ facility at the NASA Langley Research Center, the subject of this paper, underwent a number of modifications during 2001-2002, including a new drive motor and a new wall pressure measurement system among various other upgrades. Specifically, the purpose of the new wall pressure measurement system is to measure more accurately the pressure signature at the walls with or without the test article. This measurement in turn is used in specifying the boundary condition for a wall interference correction code. Corrections to obtain the equivalent free-air conditions are therefore computed based on boundary measurements and force measurements. The general experience is that this leads to a more accurate estimation compared to the classical corrections based on lift and drag only. Of course, this claim needs to be substantiated with extensive cross-tunnel validation tests using different size models.

This paper is a description of the improvements in wall correction estimation using the new wall pressure system at the $14 \times 22-\mathrm{Ft}$ facility. Section 2 describes the new wall pressure measurement system in more detail; detailed analysis of the quality of these wall measurements is given in Reference 2. This is followed by Section 3 which presents wall interference basics, terminology and definition of common terms used. The classical methods used previously at the facility are discussed in Section 4. The wall signature method is then introduced in Section 5 with a detailed description of the TWICS wall signature method now implemented in the tunnel. Sections 6 and 7 discuss the two important parts of the TWICS implementation, viz., the pre-computed perturbation velocity database (PVD) and the empty tunnel calibration database used as a tare in estimating the incremental wall signature for a specific test point. Sections 8 and 9 present some results from a recent semispan and a full-span test at the facility. As an example of improvements made on the basic TWICS method, the issue of deflected wake and its effect on the wall correction is presented in Section 10. Finally, a brief summary and concluding remarks are given in Section 11.

\footnotetext{
* Senior Member, AIAA; Aerodynamics Section Supervisor, Langley Program Office.

${ }^{\dagger}$ Member, AIAA; Aeronautical Engineer, Langley Program Office.

${ }^{\ddagger}$ Student Member, AIAA; Research Engineer, Research Facilities Branch, AAAC.
}

Copyright $(2003$ by the American Institute of Aeronautics and Astronautics, Inc. The U.S. Government has a royalty-free license to exercise all rights under the copyright claimed herein for Government purposes. All other rights are reserved by the copyright owner. 


\section{THE NEW 14x22-FT TUNNEL WALL SYSTEM}

The $14 \times 22-F t$ tunnel underwent extensive modifications in the Fall 2001 to Spring 2002 time period. A new main drive motor and a new boundary-layer suction grating were installed during this time in addition to various tunnel control and data acquisition upgrades. A new wall pressure measurement system was also put in place. Prior to the upgrade, wall pressures were measured in the center of the two sidewalls (called the North and South walls) and in the center of the ceiling along a row of 30 wall port locations distributed along the 40-ft test section length. However, the quality of wall pressure measurements was poor due to inferior measurement instrument accuracy, poor surface and orifice quality and possibly due to various leakage flows into the test section ${ }^{3}$.

The new wall pressure measurement layout is shown in Figure 1. It consists of 12 rows of 31 wall pressure taps (4 rows in each of the south, ceiling and north walls; see Figure 1 for wall and row designation convention). The wall port distribution density in the odd-numbered rows is selected such that a higher resolution is obtained at the front model cart center location of 17.75 $\mathrm{ft}$. The port distribution in the even-numbered rows is weighted such that a higher resolution is obtained at an alternate downstream model center location. The wall orifices are drilled into plates which are affixed to the wall in $30 \mathrm{ft}$ segments. Various gaps in the walls were plugged or sealed to reduce leakage.

The pressure lines are connected to electronically scanned pressure (ESP) modules which are supplied a reference pressure from a pressure calibrator unit (PCU). The ESP modules have a full scale range of 10 inches of water $( \pm 0.361 \mathrm{psi})$. Since the tunnel dynamic pressure $(Q)$ can be as high as $0.95 \mathrm{psi}$, the ESP module reference pressure is set depending on the tunnel $Q$ so that the wall ESP pressure differential is well within its stated range. Wall measurements are thus made in a smaller range tuned to the tunnel $Q$ resulting in improvements in measurement accuracy.

\section{OVERVIEW OF TUNNEL WALL CORRECTIONS}

Wall interference refers to the changes in the measured tunnel-stream reference conditions and model parameters due to the constraining effects of the tunnel walls. In the case of solid walls, the natural outward expansion of the streamlines is prevented, causing the flow about the model to accelerate over that in free air.
Open jet boundaries allow the flow to over-expand, causing the flow at the boundary to slow and balloon outward. The flow at a ventilated wall is somewhere between these two extremes. These changes in the boundary conditions can make the tunnel flow around the model substantially different from the free-air flow. The tunnel measured values reflect this changed flow, and corrections must be applied to the measured and derived values to get the equivalent free-air values (i.e., when the walls are removed). References 4 and 5 give a comprehensive review on the topic of wall interference.

To illustrate the idea further consider a threedimensional point source in free-stream simulating a Rankine forebody. The free-air solution is given by the sum of the free-stream potential and the source potential. The perturbation velocities in free-air are simply the derivatives of the source potential. When this singularity is enclosed by four solid walls, flow tangency is imposed at the boundaries which is obtained by placing an infinite number of singularities at reflection locations off the walls, following the wellknown method of images. Therefore, the additional perturbation potential introduced by the walls is the sum of all the image potentials. Correction for wall interference thus corresponds to the sum of reflection potentials, which can be evaluated once the original singularity strength is known.

Wall interference correction is thus a spatially varying function. The traditional assumption is that the perturbation velocity field can be approximated by a change in the angle of attack and the tunnel velocity. Any left-over differences are usually second-order effects. However, when they become significantly large such as in the case of a large model, the measured data may become uncorrectable by traditional methods. A CFD solution of the in-tunnel flow is then required. This is however costly for routine use at a wind tunnel facility.

The perturbation velocity field is usually computed using a wall signature method in which a simplified representation of the model is made using potential singularity elements such as point sinks, point sources, and point or line doublets. Once the perturbation velocity solution based on the measured boundary condition imposed is known, wall corrections can be quantified by averaging the interference flow field along model or tunnel reference lines. The corrections, termed primary or mean wall interference corrections, are given in terms of a blockage correction $\varepsilon$ and an angle of attack correction $\Delta \alpha$ 
The blockage correction $\varepsilon$ is obtained as the average of streamwise perturbation velocities (normalized by tunnel reference velocity) along the model axis. This is proportional to the ratio of the maximum model frontal cross section area to the tunnel cross section area. This correction is applied to the measured values of Mach number $M$ and dynamic pressure $Q$. The corrections for $M$ and $Q$ are obtained as

$$
\begin{aligned}
& \frac{\Delta M}{M}=\left(1+\frac{\gamma-1}{2} M^{2}\right) \varepsilon \\
& \frac{\Delta Q}{Q}=\left(2-M^{2}\right) \varepsilon
\end{aligned}
$$

The angle of attack correction is obtained as a weighted average of the perturbation velocity in the lifting direction. The wing $3 / 4$ chord line is the reference line customarily used for this averaging, although some classical methods prefer the $1 / 4$ chord line. Weighting is done based on the spanwise load distribution. These primary corrections lead to corresponding corrections on force coefficients $C_{L}$ and $C_{D}$. A buoyancy correction on $C_{D}$ due to wall interference is also computed from the perturbation velocity gradient averaged along the model axis. Pitching moment coefficient corrections are computed based on changes in flow curvature calculated from spatial variations in the angle of attack corrections.

The interference flow field in the vicinity of the model is also of interest in order to assess the extent of change in mean corrections with change in reference lines or points where they are averaged. These local values of corrections are presented as contour plots at different tunnel sections.

To summarize, wall corrections for a test point are reported as follows:

1. A blockage parameter, $\varepsilon$ and an angle of attack correction, $\Delta \alpha$; these are the primary corrections obtained by averaging the perturbation flow field.

2. Corrections on tunnel Mach number and dynamic pressure, which are functions of the blockage parameter, $\varepsilon$.

3. Corrections on lift, drag and moment coefficients which result from the changed tunnel reference velocity, angle of attack and flow gradients. These are derived corrections obtained from the perturbation flow field.

4. Contour plots of perturbation velocity field in the model vicinity; these show the extent of the departure of local corrections from the averaged correction values.

The free-air or corrected values are obtained by adding the corrections to the measured values. For a lifting model in a solid-walled tunnel, the free-air or corrected values of $M, Q, \alpha, C_{D}$ are larger than the measured values; free-air $C_{L}$ is usually decreased. Depending on the model and the test, corrections on roll moment, sideforce and tail incidence can also be calculated.

\section{EXISTING METHODS AT THE 14x22-FT TUNNEL}

The existing wall corrections used in the facility rely on standard formulae based on model and tunnel geometry, measured lift and drag values. This 'classical' blockage correction is documented in References $4,6,7$ and various other references dating back to 1960 . It consists of a fuselage volume blockage term, a wing blockage term and an attached wake blockage term based on the apparent drag coefficient. The jet boundary correction (equivalent to an angle of attack correction) is also applied based on the lift and induced drag coefficients. While this method is of acceptable accuracy for a small model in attached flow conditions, large departures are possible when dealing with large models at high-lift conditions. For models with a large deflected wake, a method based on Heyson's work ${ }^{8}$ is applied to calculate the change in the angle of attack and dynamic pressure in the model region.

Unfortunately, many questions exist regarding the validity of the assumptions required for derivation of these classical methods. In addition, no assessment of correction uncertainty is available. Improving the accuracy of wall corrections involves the use of the measured wall-pressure boundary condition, which incorporates a more realistic characterization of the intunnel flow into the correction method and hence a tighter control on the accuracy of corrections.

\section{THE TWICS WALL SIGNATURE METHOD}

The Transonic Wall Interference Correction System (or TWICS) is a wall correction code based on the wall signature method developed for transonic tunnels with ventilated walls, originally for the Ames 11-Ft Tunnel ${ }^{9}$. It has been implemented for the $14 \times 22-\mathrm{Ft}$ tunnel in the solid-wall or 'walls down' configuration. TWICS and its predecessor code WICS $^{10}$ were developed by Ulbrich by using a strategy of globally fitting the wall signature. A brief summary of the method is given below. 
TWICS uses the pressure signature at the walls (actually the incremental value relative to tunnel empty signature) as the basis for computing wall interference corrections. The model is represented by a number of point doublets (to simulate volume and wake blockage) and line doublets (to simulate effects due to lift). The far-field effect due to the assumed singularity distribution is matched with the wall signature. This is done in a global fitting procedure, which yields the strengths of the singularities as the solution. The perturbation velocities are then computed based on superposition of standard solutions of point and line doublets which are contained in pre-computed databases of perturbation velocity solutions. Corrections for each test point are obtained by interpolation from the database at near-real time speeds. Compressibility is modeled using Prandtl-Glauert scaling. As a result, there is an upper limit to the Mach number in the application of this method.

The TWICS code is designed to work in unison with a panel-method-generated database of wall interference solutions based on point doublets for blockage and line doublets for lift interference. By appropriately setting the boundary conditions in the panel method, ventilated walls can also be modeled. Since the $14 \times 22$ tunnel is operated as a solid-wall facility, this feature of TWICS is not used. A simpler procedure based on the method of images is used to generate the database.

The string of point doublets used in the TWICS method to model volume and wake blockages can be shown to be equivalent to the source-sink pair used in the WICS method. The advantage is that the individual point doublet strengths can now be weighted in proportion to the cross section area of the test article thereby increasing the model fidelity. In TWICS, the effect of the sting is also modeled using a chain of weighted point doublets, in an analogous fashion to volume blockage. This shortcut implies the use of a considerably smaller tunnel calibration test matrix.

The inputs for TWICS are described below:

1. Tunnel empty signature: The wall signature is defined in terms of the 12 rows with 30 orifices in each. This calibration data is required for a specified range of Mach numbers (or equivalently, dynamic pressures). For full span models, the signature with the model support (which is the vertical post at $X=40 \mathrm{ft}$ ) at several pitch angles is also required at various operating conditions. Several such calibration sets are required depending on the support system used and the floor boundary layer suction system (BLRS) state (on or off). See Section 7 for details of the tunnel calibration.
2. Wall signature for a given test point.

3. Test point values of uncorrected force and moment values, Mach number, reference velocity at model center of rotation and a number of other test and model attitude parameters.

4. Perturbation velocity database (PVD): This is a large table of pre-computed perturbation velocities used in signature matching and wall interference computation. The database depends on the wall orifices layout, tunnel section, Mach number and lift vector direction. See Section 6 for details of generating these databases.

5. Model singularity distribution and geometry data.

6. Reference lines along which weighted averages of interference are to be computed and planes along which local values of wall interference are to be computed.

7. Port flags used to de-select specific wall orifices that are not to be used in the calculation for a given test. In addition to this, the code rejects additional wall orifices based on statistics of the fit (see Reference 10 for the original rejection criteria). Here an improved rejection criteria is used based on wall data quality analysis (see Reference 2 for details). Hence the original statistical rejection logic used in the code is turned off.

The calculation steps used in TWICS are described below:

1. Processing of input test data: For each test point, the wall signature is read in. Subsequently, the corresponding tunnel empty signature is interpolated from the calibration database and subtracted to get the incremental or 'tared' wall signature.

2. Computation of the equivalent line doublet strength from measured lift and model geometry: The strength is then distributed along the span as per input or computed weights. These weights are based on the estimated wing loading distribution.

3. Interpolation from the perturbation velocity database (PVD): This is done to estimate the lifting effect part of the signature at each port. The lifting part of the signature is then subtracted from the tared signature to get the blockage effect at each port.

4. Least squares fitting and interpolation from PVD to calculate the strengths of the point doublets: The two unknowns computed here are the volume blockage strength and the wake blockage strength. This step represents the core of the calculation procedure.

5. Interpolation from PVD to compute wall interference at any point in the test section (within reference grid limits) by superposition of all singularities: Mean corrections are then calculated using weighted averaging. Force and moment coefficient corrections are then computed. The 
streamwise distribution of blockage is used to estimate the buoyancy correction.

6. Iteration using corrected tunnel parameters, if necessary.

Corrections are computed for each point in a polar independently. The primary mean correction due to blockage is applied as corrections on Mach number, $M$ and dynamic pressure, $Q$ (added to corresponding measured values). Upwash correction is applied as correction on the angle of attack. Corrections on $C_{L}, C_{D}$ and pitching moment coefficient $C_{M}$ are computed based on the primary mean corrections of blockage and angle of attack. In addition, model-induced buoyancy correction is also calculated and added to $\Delta C_{D}$. The method also computes local variations of interference corrections, which are useful in determining if the averaging assumption is truly representative of the interference field in the model region.

\section{PRE-COMPUTED PERTURBATION VELOCITY DATABASES}

The databases for TWICS are large tables of precomputed solutions of perturbation velocities induced by unit singularities (point doublets or line doublets) enclosed by the tunnel walls. In these database runs, the singularities are placed at different locations in the tunnel (termed the singularity grid) and solutions are calculated at different locations inside the tunnel (termed the reference grid) as well as at the wall port locations.

The database table is thus a function of a number of variables as given below.

Type of singularity: The point doublet singularity is the basic building block for solid or wake blockage due to a model in the tunnel. This is the same as a point source plus a point sink in close proximity. The line doublet singularity is the building block for lift interference in the tunnel. This is equivalent to a chain of point doublets starting at the line doublet location stretching to infinity.

Orientation of singularity: Point doublet direction used here is always pointing in the upstream direction (i.e., point sink to point source direction is $-X)$. Line doublet direction is opposite to the lift vector direction (i.e., chain of point doublets pointing away from the lift direction). In order to handle a case with model roll where lift direction changes, calculations are done at $\mathbf{4 5}$ degree intervals of the line doublet angle in order to facilitate interpolations.
Singularity location: The location is determined by a specified singularity grid; the model and support system should be contained within this grid.

Solution location: The location is specified by a reference grid. Reference lines along which wall corrections are averaged (such as fuselage centerline, wing $3 / 4$ chord line) should be contained within this grid. Solutions are also computed at all pressure measurement locations on the tunnel walls. The solution at a tunnel interior point consists of the three perturbation velocity components $(u, v, w)$; the solution at the wall consists of only the streamwise component $u$.

Model configuration: The database depends on whether the model configuration is semispan or fullspan. The location of the singularity and reference grids are dependent on the model configuration. The solution also depends on the model configuration since the semispan model-mounting wall becomes an image plane with the effective tunnel section doubling in size. Mach number: With the use of the Prandtl-Glauert scaling factor $\beta=\sqrt{1-M^{2}}$, the effective tunnel cross section is reduced by this factor. The singularity strengths also scale with this factor $\left(\beta^{2}\right.$ for point doublet, $\beta^{3}$ for line doublet).

Note also that the solutions calculated at the wall include the direct effect of the singularity. This can then be compared to the wall signature. The solutions calculated at the interior of the tunnel do not include the direct effect of the singularity (it is thus equivalent to the summation of solutions from only the images of the singularity which is the true definition of the interference solution).

In TWICS, once the actual location of a singularity in the tunnel is established, multi-parameter linear interpolation is used to find the corresponding perturbation velocity solution. For wall locations, the actual coordinates of the wall ports are used in the database. A master database is first generated for all the wall ports; a customized database for a given test is then derived from it depending on the ports actually selected for use in a particular TWICS run.

\section{TUNNEL CALIBRATION DATA-BASES}

Measuring the tunnel-empty wall signatures periodically is a recommended quality control measure for the wall system. It is also required in TWICS to subtract out systematic variations from orifice to orifice and the effect due to the tunnel boundary layer. The assumption made here is that these effects are constant 
with or without the model in the tunnel. The calibration is done for a number of $Q$ values, so that interpolation can be done for the test-point value of $Q$. For full-span models, the model sting is also considered in the TWICS simulation, so there is no need to obtain a calibration with the sting or other support components which are aligned with the wake. However, other parts of the support system (such as the vertical post for the $14 \times 22$ tunnel) should be included in the calibration. If the geometry of these components change with angle of attack, for example, then the calibration should be done in a range of such parameters. Interpolation for the tunnel empty signature will then be a multi-parameter one involving for example, $Q$ and the angle of attack. Separate databases are required with floor boundary layer removal system on and off.

\section{EXAMPLE RESULTS: SEMISPAN MODEL}

Wall corrections for a large semispan wing tested recently at the facility will now be discussed. The model is a trapezoidal planform high-lift wing with fixed-setting flaps and slats. A photo of the model installed in the tunnel is shown in Figure 2. Some of the important model and tunnel geometry parameters and flow conditions are given in the following table.

\begin{tabular}{|l|l|}
\hline Wing area & $22.028 \mathrm{sq} \mathrm{ft}$. \\
\hline $\begin{array}{l}\text { Wing } 1 / 4 \text { chord, } 1 / 2 \\
\text { chord sweeps }\end{array}$ & $30^{\circ}, 26.1^{\circ}$ \\
\hline $\begin{array}{l}\text { Root, tip, aero. and } \\
\text { geom. chords }\end{array}$ & $\begin{array}{l}4.6,1.7,3.3, \\
3.1 \mathrm{ft}\end{array}$ \\
\hline Half span, aspect ratio & $7.088 \mathrm{ft}, 4.561$ \\
\hline Tunnel cross section & $\begin{array}{l}14.75 \mathrm{by} 21.75 \\
\mathrm{ft}\end{array}$ \\
\hline $\begin{array}{l}\text { Model center of } \\
\text { rotation }\end{array}$ & $\begin{array}{l}X=17.75, \\
Y=-7.25 \mathrm{ft}\end{array}$ \\
\hline Tunnel $Q$ & $57.8 \mathrm{psf}$ \\
\hline $\begin{array}{l}\text { Mach number, } \\
\text { velocity }\end{array}$ & $0.2,230 \mathrm{fps}$ \\
\hline Angle of attack & -4 to $36^{\circ}$ \\
\hline
\end{tabular}

The test was run with the BLRS on. All test runs were made at a fixed unit Reynolds number of 1.4 million. The $C_{L}$ vs. $C_{D}$ curve for this wing model is shown in Figure 3, which is typical of a high-lift configuration. Stall angle is 34 to $35^{\circ}$ with a maximum $C_{L}$ of 3.0 and a $C_{D \text {, stall }}$ of 0.7 .

The singularity distribution is specified a priori with a number of $-X$ direction point doublets along the fuselage axis and wake separation lines. Line doublets originating from the $1 / 4$ chord line are used to model the lifting effect with an elliptic loading distribution.
Figures $4 a$ and $4 b$ show the distribution used in TWICS. The specified locations correspond to zero angle of attack; for a particular data point, these locations are displaced as a function of the model kinematics and attitude ( $\alpha$, in the present case).

Wall correction results for a representative run (a polar of 28 points from $-4^{\circ}$ to $35^{\circ}$ ) is given here. Figure 5 shows the mean corrections for this run. The correction on the blockage parameter $\varepsilon$ is shown as well as the corrections on $Q$. The blockage stays below the $0.25 \%$ value for angles of attack below $25^{\circ}$. Between $25^{\circ}$ and $34^{\circ}$, blockage increases to double this level $(0.5 \%)$ due to a larger contribution from the wake. Above $34^{\circ}$, the wing begins to stall and the large separated wake increases the blockage even further. Also shown in the plot of $\Delta Q$ are the correction values calculated by the existing classical procedure as well as using the Maskell procedure ${ }^{11}$. The two-step form of the Maskell method estimates the separated flow effects on blockage using a formulation based on flat plate flow measurements. The blockage predicted by TWICS falls in between the classical and Maskell results.

The angle of attack correction shown in Figure 5 is largely a function of lift and follows the $C_{L}$ vs. $\alpha$ shape. A maximum $\alpha$ correction of $1.6^{\circ}$ is obtained at $\alpha=32^{\circ}$ which means the equivalent free-air angle of attack is $33.6^{\circ}$. In TWICS, the $\alpha$ correction is obtained by averaging at the $3 / 4$ chord line. The classical values obtained by the jet boundary correction of Reference 6 , also shown in Figure 5, seem to be more in agreement with the TWICS corrections obtained by averaging at the $1 / 4$ chord line.

Corrections on the force and moment coefficients are shown in Figure 6. The $\Delta C_{D}$ and $\Delta C_{L}$ values from the classical approach are also shown to be in close agreement, with a slight departure only near stall conditions. The buoyancy correction on $C_{D}$ is shown to be quite small, varying linearly with the angle of attack to a maximum value of 25 drag counts. The changed aerodynamic characteristic due the wall interference in the form of a corrected $C_{L}-C_{D}$ curve is also shown in Figure 6. Overall, as seen from Figures 5-6, the wall corrections are substantial and need to be considered when evaluating the free-air performance of the wing.

A good way to verify how the TWICS model simulates the real flow is to compare the measured incremental wall signature with the TWICS prediction. Shown in 
Figure 7 are the incremental wall signatures along the 12 rows compared with the TWICS values for 3 angles of attack. The overall agreement is remarkably good, except at Rows 1 and 12 at high angles of attack. Some of this difference is due to a deflected wake which tracks closer the north wall and is not simulated in TWICS. Section 10 provides more details on capturing this effect which leads to a better agreement. It is also possible that the simple lift model used in TWICS is also responsible for part of this difference in the signatures.

\section{EXAMPLE RESULTS: FULL-SPAN MODEL}

Wall corrections for a relatively small full span wing (reference area only $2 \%$ of tunnel cross section, compared to $7 \%$ for the trapezoidal wing) will now be presented. The wing is an elliptical planform wing with symmetric NACA 0012 sections. This is a more limiting test case for the wall pressure system in that the wall signatures are much weaker (only $10 \%$ of the trapezoidal wing signatures for the same angle of attack). Some of the important model parameters and flow conditions are given in the following table.

\begin{tabular}{|l|l|}
\hline Wing area & $6.25 \mathrm{sq} \mathrm{ft}$. \\
\hline Wing sweep & $0^{\circ}$ \\
\hline Root chord & $1.2 \mathrm{ft}$ \\
\hline Span, aspect ratio & $6.75 \mathrm{ft}, 5.625$ \\
\hline Model length & $2.8 \mathrm{ft}$ \\
\hline Model center of & $X=17.75, Y=0$ \\
rotation & $\mathrm{ft}$ \\
\hline Tunnel $Q$ & $2-137 \mathrm{psf}$ \\
\hline Mach number, & $0.05-0.32$, \\
velocity & $6-350 \mathrm{fps}$ \\
\hline Angle of attack & -3 to $12^{\circ}$ \\
\hline
\end{tabular}

A photo of the model installed in the tunnel along with the support system is shown in Figure 8. The support system consists of a sting, a roll coupling, a long sting ('cannon'), and a vertical post. The support system moves in the pitch plane with the model fixed at the $X=17.75, \quad Y=Z=0$ position. The aerodynamic characteristic of the model is shown in Figure 9.

A tunnel-empty calibration with the support system alone installed in the tunnel with repeat runs at several $Q$ and $\alpha$ values is used here. Generally, an empty tunnel calibration can also be used without any of the wake-oriented support system components (these can be modeled in TWICS). However, the vertical post should be part of the empty tunnel calibration. For the present case, the only good calibration set that is available is the one with the complete support system in the tunnel. This essentially means that the singularity distribution used here will correspond to only the model and wake and not any of the sting components.

The singularity distribution used for the elliptical wing model is shown in Figure 10 . Wall correction calculations were done for a number of $Q$ values in the $\alpha$ range $-3^{\circ}$ to $12^{\circ}$. Results indicate that the blockage is nominally constant across this range at 0.001 . It was also found that for $Q$ values less than $10 \mathrm{psf}$, the scatter in the computed blockage values is large, due to the wall system not able to distinguish the weak signature from other random variations. The corrections on $M$ and $Q$ are also constant in the $\alpha$ range and they scale with $M$ and $Q$ as given by Equation (1) and (2) ( $\Delta M / M=\varepsilon ; \Delta Q / Q=2 \varepsilon$, approximately). The angle of attack correction scales with $\alpha$ with $\Delta \alpha / \alpha=0.011$, approximately. The classical value calculated from charts in Reference 7 is also 0.011 .

The corrections on the force coefficients are shown in Figure 11. The correction on drag reaches a maximum of 20 drag counts at $12^{\circ}$ angle of attack; corrections on lift and drag due to buoyancy are negligibly small.

Comparison of measured incremental wall signatures with TWICS-fit values at $\alpha=-3^{\circ}, 2^{\circ}$ and $12^{\circ}$ is shown in Figure 12. A good global fit has been obtained even at these low wall signature values. Note that the wall signature scale is \pm 0.01 compared to the \pm 0.1 scale used for the trapezoidal wing case in Figure 7.

\section{DEFLECTED WAKE ANALYSIS}

The standard approach used in classical wall correction methods as well as in wall signature methods such as TWICS is to model wall-induced lift interference using a number of line doublets distributed along the wing span with starting locations along the $1 / 4$ chord line. Each line doublet is also equivalent to a semi-infinite streamwise string of point doublets, pointing opposite to the lift vector direction, and originating from the same $1 / 4$ chord locations of the wing. For a threedimensional wing there will be a number of such point doublet strings at different span locations.

For high-lift wings as well as rotorcraft in forward flight, the vorticity in the wake is oriented at an angle to the freestream in an average sense. In addition, the corresponding point doublet vectors are inclined to the free-stream at an angle primarily dependent on the liftdrag ratio, as modeled by Heyson'. Heyson's wake deflection model thus assumes a straight-line trajectory for this vorticity track. This model is equivalent to a 
semi-infinite string of point doublets placed along a deflected wake line, with the point doublet direction at an angle to the lift vector direction as shown in the sketch in Figure 13a.

The Heyson model calculates the deflected wake wall interference velocities in two steps. First the effective wake deflection angle is calculated. This is a function of the wing span, area, lift, induced drag, tunnel geometry and free-stream velocity. The second step in the Heyson model is to calculate the singularity strengths and corresponding interference velocities. As shown Figure 13b for the trapezoidal wing case, the point doublets are split into two types, one pointing to $-Y$ producing the lift effect and another pointing to $-X$ producing the induced drag effect.

Since the results from the first step of the Heyson analysis revealed that the vorticity may be deflected by as much as $10^{\circ}$ on the average from the chord plane at maximum lift condition, a modified version of the TWICS code was created to include these effects. In order to implement the deflected wake, the line doublets used in TWICS were changed to $-Y$ direction point doublet strings noting that a summation of a chain of these point doublets is equivalent to the line doublet with a $90^{\circ}$ orientation. It is then possible to deflect the vorticity track by specifying the point doublets to be located along the deflected line. This requires the generation of a new PVD database with interference solutions due to $-Y$ direction point doublets. The first part of the Heyson model was added to TWICS to first compute the effective wake deflection angle. Changes were made in the interpolation scheme in TWICS to locate the deflected lifting point doublets in the database grid followed by interpolation and summation. Changes were also made to deflect the wake blockage point doublets by a corresponding angle; this effect was however found to be minor.

Figure 14 shows the wall signature fit from the deflected wake version of TWICS for $\alpha=32^{\circ}$ (for rows 1 and 12 , most affected by the deflected wake), which can be compared directly with Figure 7 from the regular TWICS calculation. It is clear that the deflected wake modification has improved the fit in these two rows of the South and North walls. Figure 15 further demonstrates that this is true for all the rows, showing that the standard deviation of the fit has decreased as a result of this modification. Note that the global standard deviation drops by a factor of almost two. Finally, Figure 16 shows the change in the mean corrections as a result of the deflected wake modification. A closer agreement with the classical values can be observed.

\section{CONCLUSION}

A wall signature-based correction method for the wallsdown configuration of the $14 \times 22-\mathrm{Ft}$ tunnel has been implemented based on a new wall pressure measurement system at this tunnel. Example results from recent semispan and full-span tests at the facility have been presented. Comparison with classical, closed-form methods of wall correction indicate that the wall signature method provides comparable values. Further, the model-provided wall signatures compare very well with measured values in a range of model sizes, angle of attack and $Q$ values. Although true validation of results require cross-tunnel tests using scaled models, it can be stated that the new method is an improvement over the existing classical methods since boundary measurements are used in addition to force and moment inputs and since model shows close agreement with the measured wall pressures.

\section{REFERENCES}

1. Hemsch, M, Grubb, J., Krieger, W., and Cler, D., "Langley Wind Tunnel Data Quality Assurance Check Standard Results", AIAA 2000-2201, June 2000.

2. Kuhl, D.D., and Everhart, J.L., "Measurement and Control of the Uncertainty of Scanning Pressure Transducer Measurements", AIAA 2003-3816, June 2003.

3. Iyer, V., and Everhart, J.L., "Application of Pressure-Based Wall Correction Methods to Two NASA Langley Wind Tunnels", AIAA 2001-2472, June 2001 .

4. Garner, H. C., Rogers, E. W. E., Acum, W. E. A., and Maskell, E. C., "Subsonic Wind Tunnel Wall Corrections", AGARDograph 109, October 1966.

5. Ewald, B. F. R. (Editor), "Wind Tunnel Wall Correction", AGARDograph 336, October 1998.

6. Quinto, P.F., and Orie, N.M., "Langley 14- by 22Foot Subsonic Tunnel Test Engineer's Data Acquisition and Reduction Manual", NASA TM 4563, June 1994.

7. Barlow, J.B., Rae, Jr., W.H., Pope, A, Low-Speed Wind Tunnel Testing, Third Ed., John Wiley \& Sons, 1999, pp. 367-427.

8. Heyson, H.H., "Linearized Theory of Wind Tunnel Jet-Boundary Corrections and Ground Effect for VTOL-STOL Aircraft", NASA TR R-124, 1962.

9. Ulbrich, N. and Boone, A.R., "Determination of the Wall Boundary Condition of the NASA Ames $11 \mathrm{ft}$ Transonic Wind Tunnel", AIAA 2001-1112.

10. Ulbrich, N., "The Real-time Wall Interference Correction System of the NASA Ames 12-foot Pressure Tunnel", NASA/CR-1998-208537, July 1998.

11. Maskell, E.C., "A Theory of the Blockage Effects on Bluff Bodies and Stalled Wings in a Closed Wind Tunnel", R\&M 3400, November 1963. 

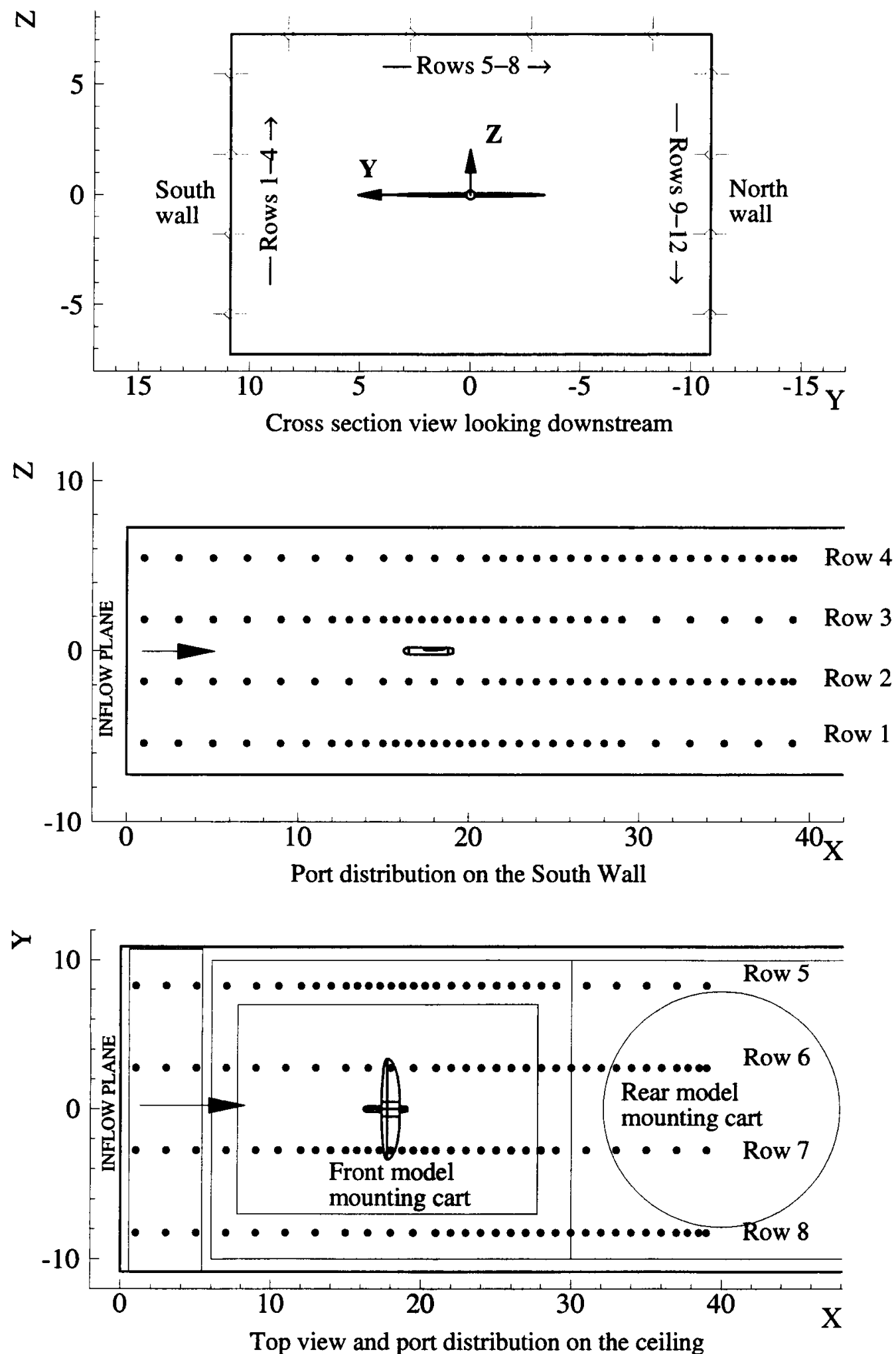

Figure 1. New wall pressure ports for the $14 \times 22-\mathrm{Ft}$ tunnel (elliptical wing model outlines shown). 


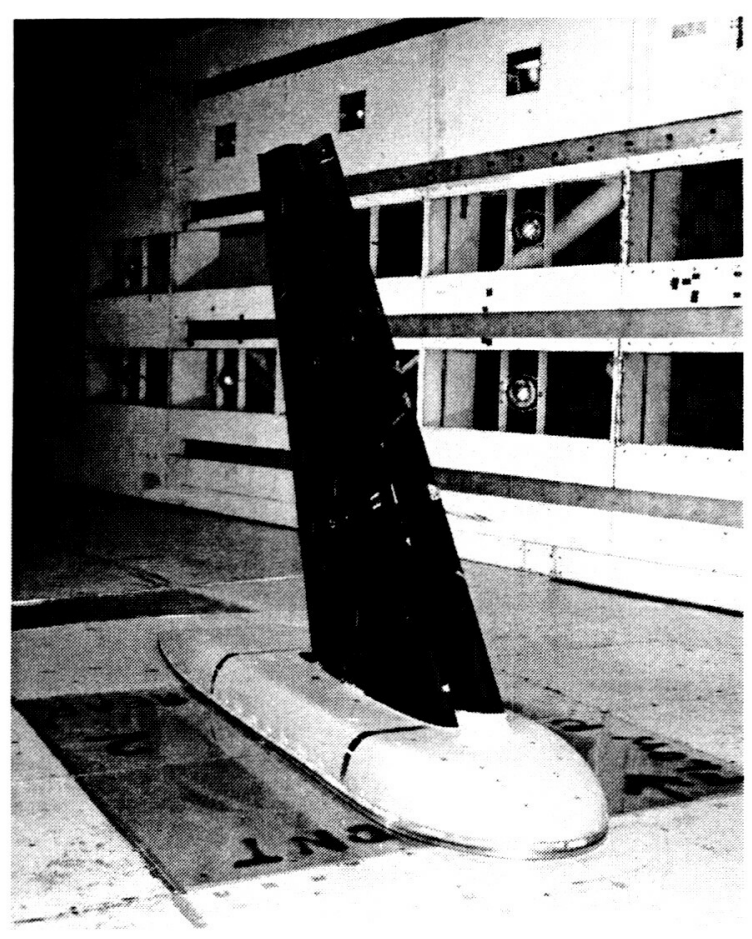

Figure 2. Photo of the trapezoidal wing mounted in the 14x22-Ft. tunnel. Photo courtesy of NASA Langley Photo Lab.

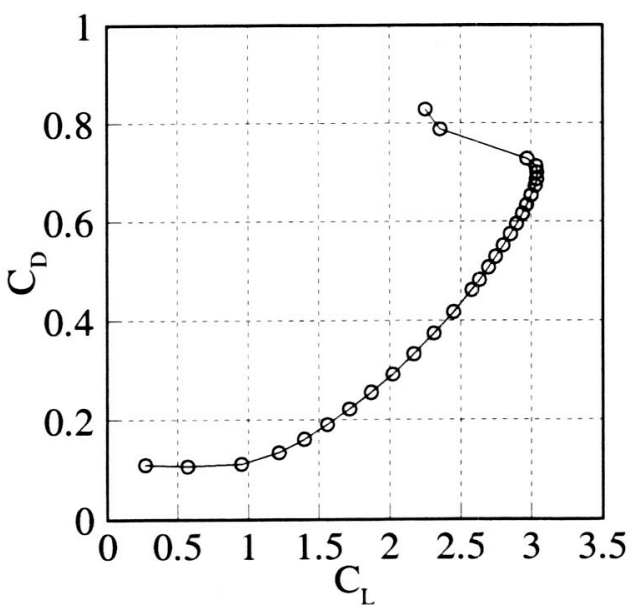

Figure 3. Aerodynamics characteristics of the trapezoidal wing.

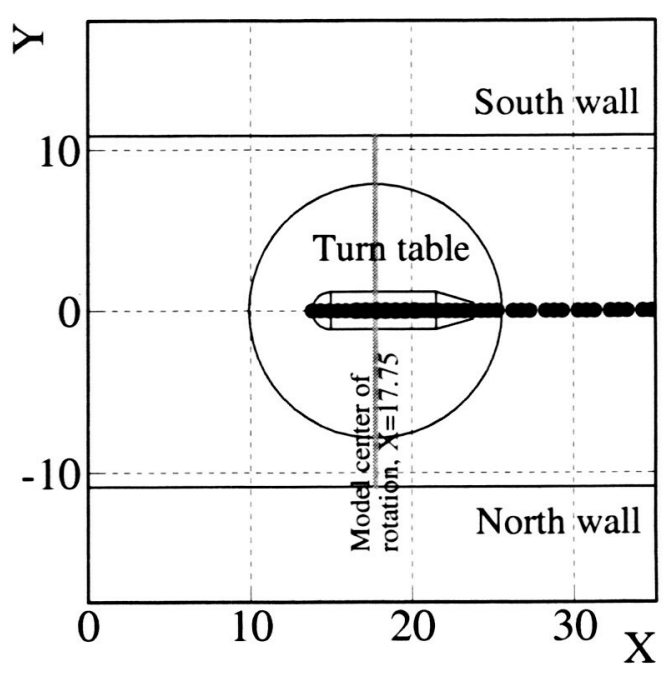

Figure 4a. Singularity distribution for trapezoidal wing, top view.

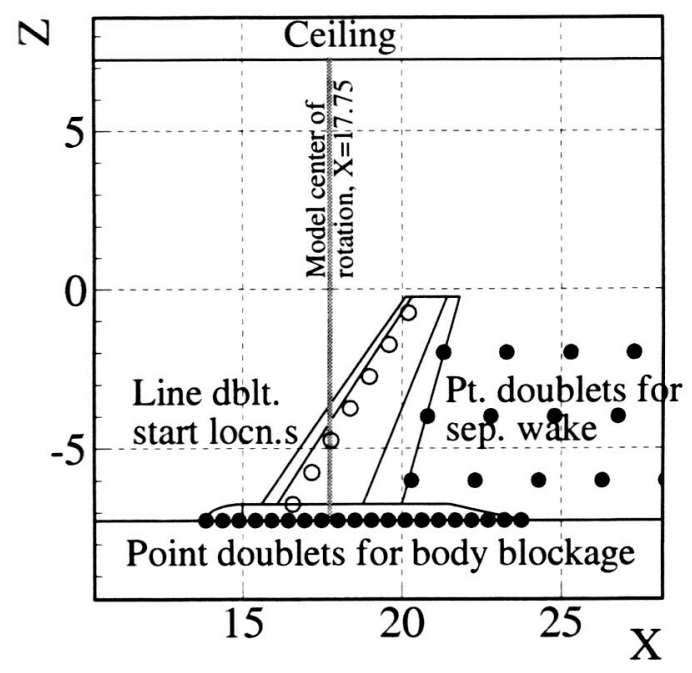

Figure $4 \mathrm{~b}$. Singularity distribution for trapezoidal wing, sideview. 

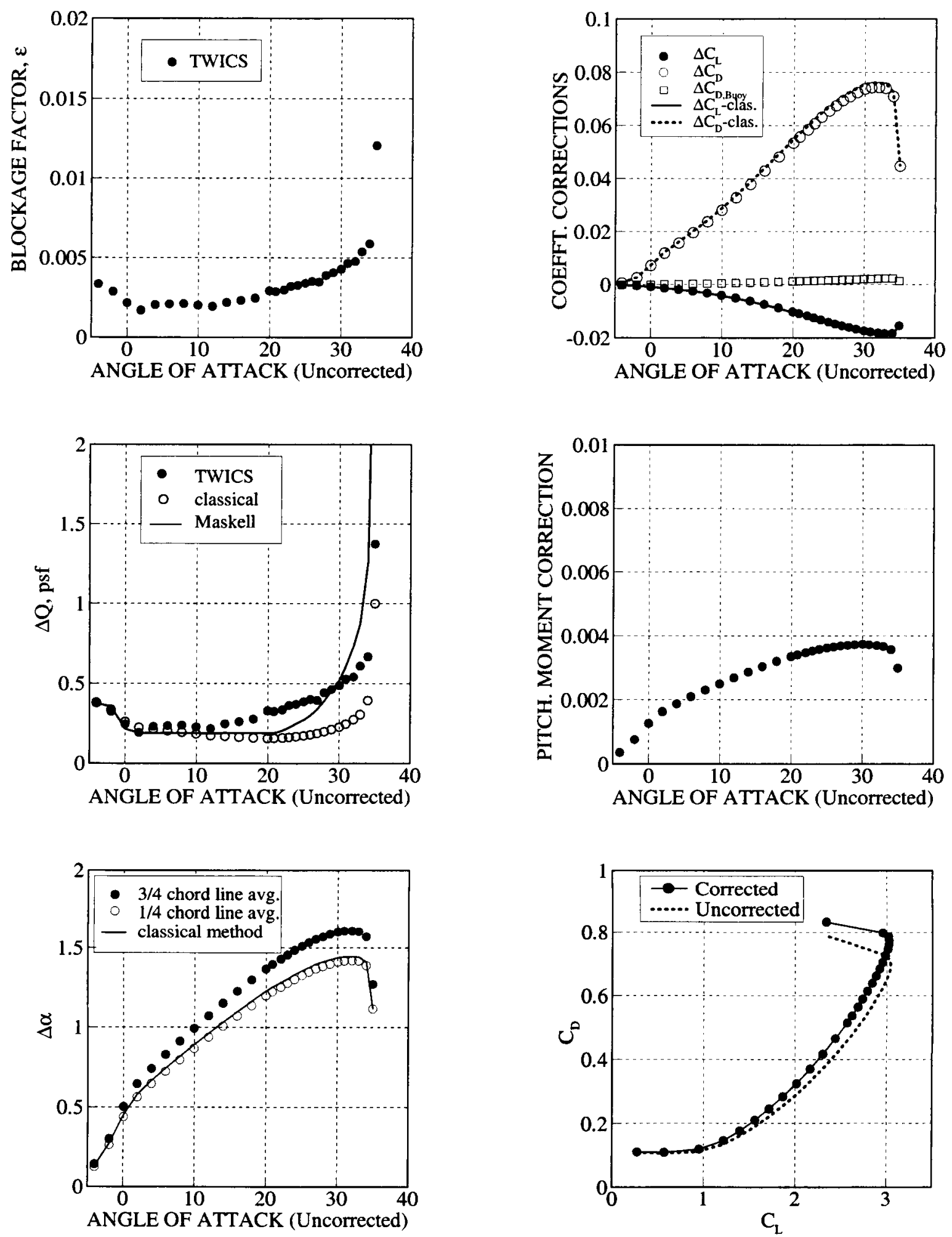

Figure 5. Mean corrections for the trapezoidal wing in the $14 \times 22-\mathrm{Ft}$ tunnel.

Figure 6. Coefficient corrections for the trapezoidal wing in the $14 \times 22-\mathrm{Ft}$ tunnel. 


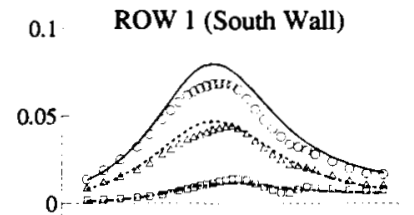

$-0.05$
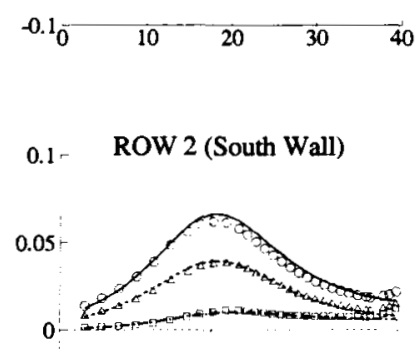

$-0.05-$
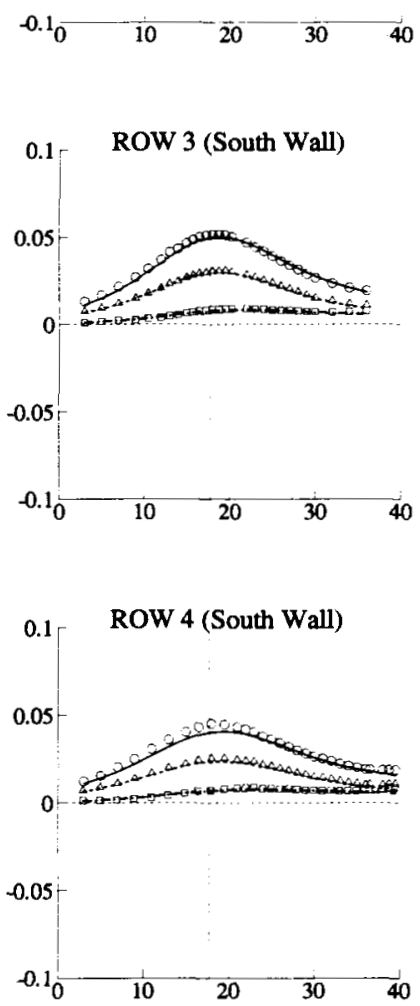
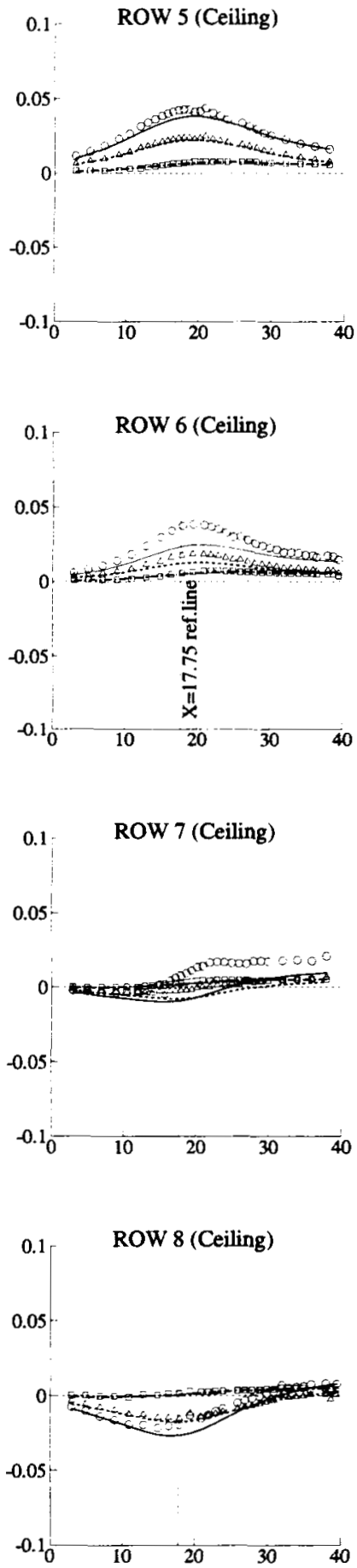

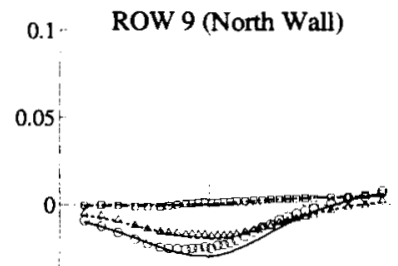

$-0.05$

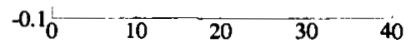

0.1- ROW 10 (North Wall)
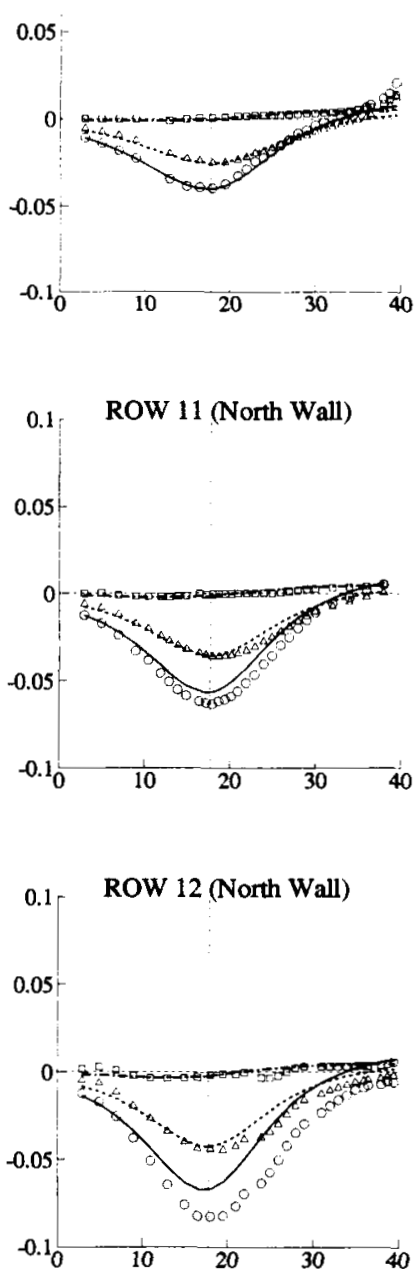

Figure 7. Measured and TWICS-fit wall signatures for the trapezoidal wing test. Symbols are incremental measurements; lines are TWICS-fit values. Three angles of attack $-^{\circ}$ (squares, dash-dot line), $10^{\circ}$ (triangles, dashed line), $32^{\circ}$ (circles, solid line) are shown. $\mathrm{X}$-axis is the streamwise distance $X ; \mathrm{Y}$-axis is the incremental normalized wall velocity. 


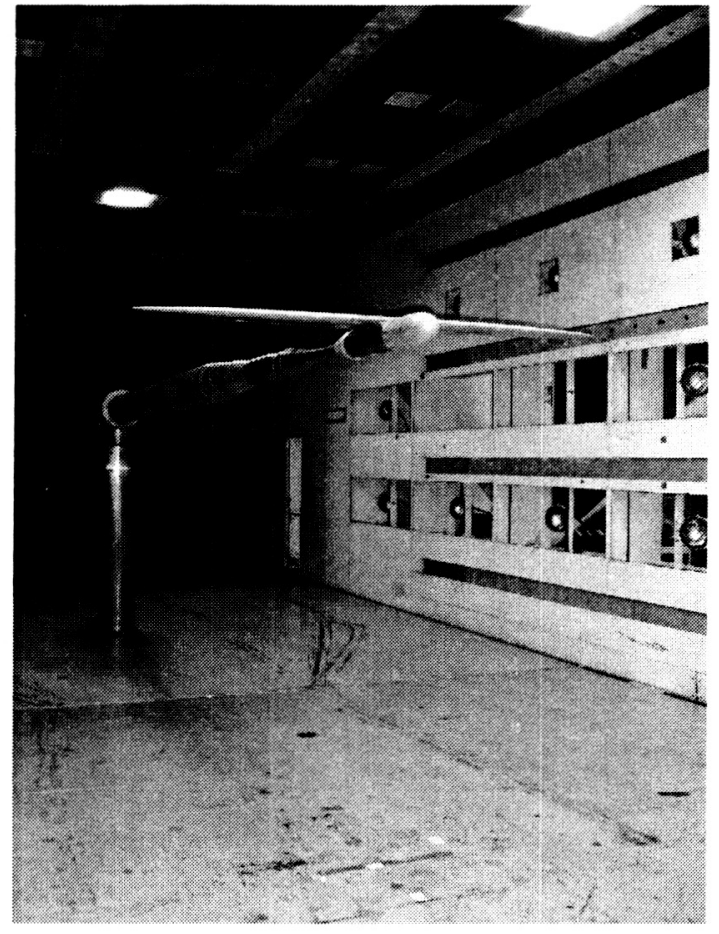

Figure 8. Photo of the elliptical wing mounted in the 14x22-Ft. tunnel. Photo courtesy of NASA Langley Photo Lab.

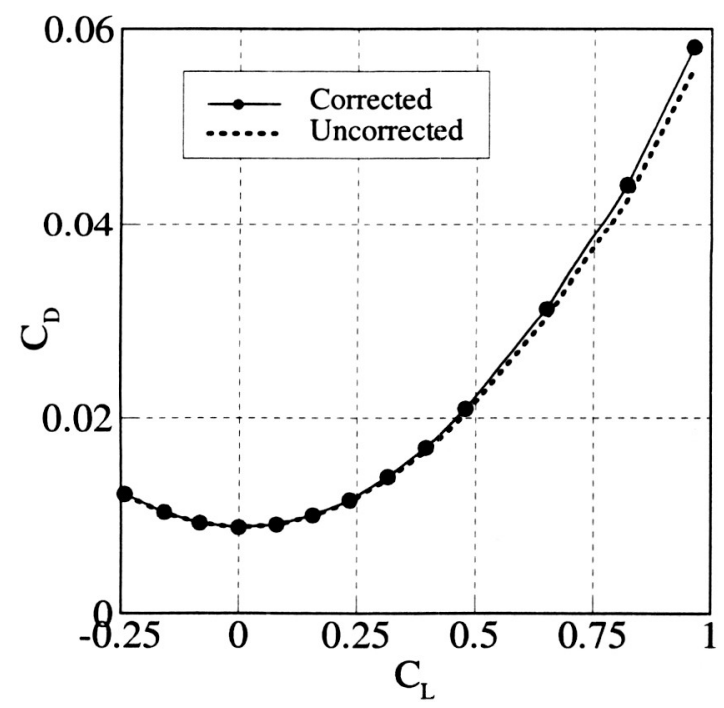

Figure 9. Aerodynamic characteristics of the elliptical wing in the $14 \times 22-\mathrm{Ft}$ tunnel.

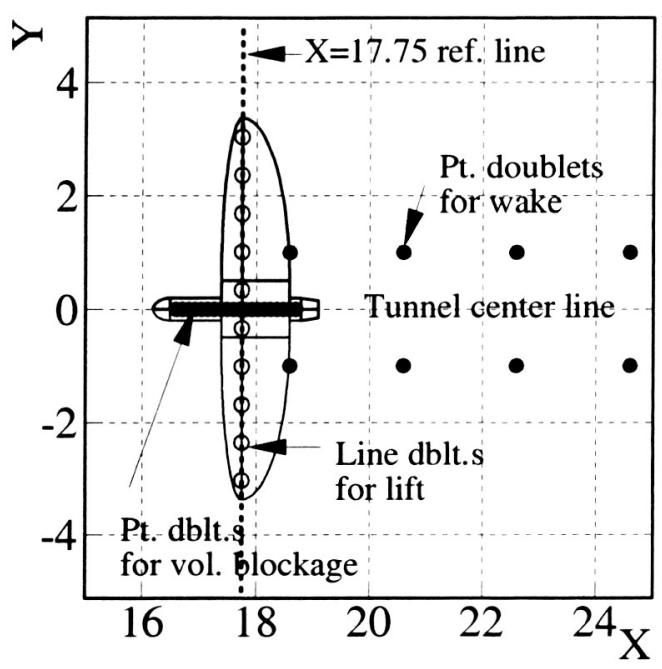

Figure 10. Singularity distribution for the elliptic wing model in the $14 \times 22-\mathrm{Ft}$ tunnel.

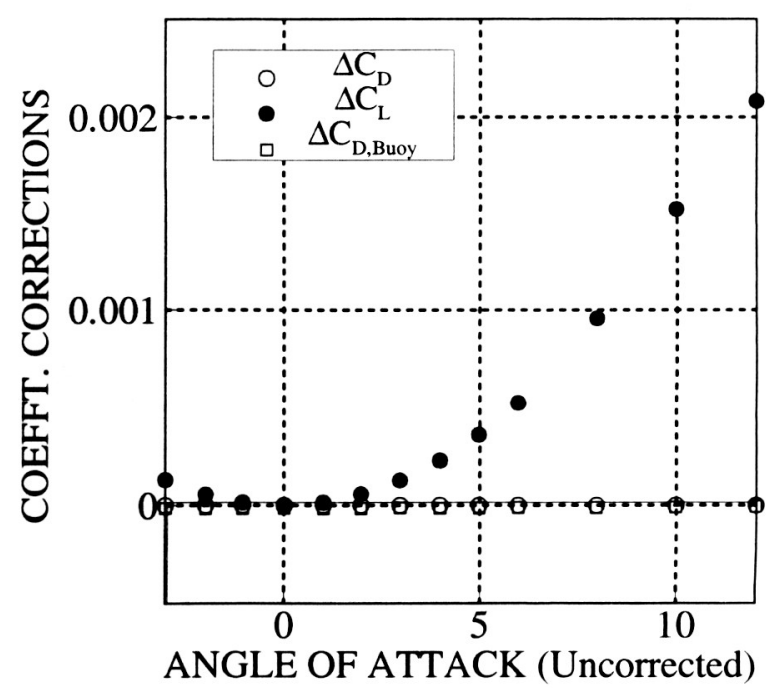

Figure 11. Coefficient corrections for the elliptical wing in the $14 \times 22-\mathrm{Ft}$ tunnel. 

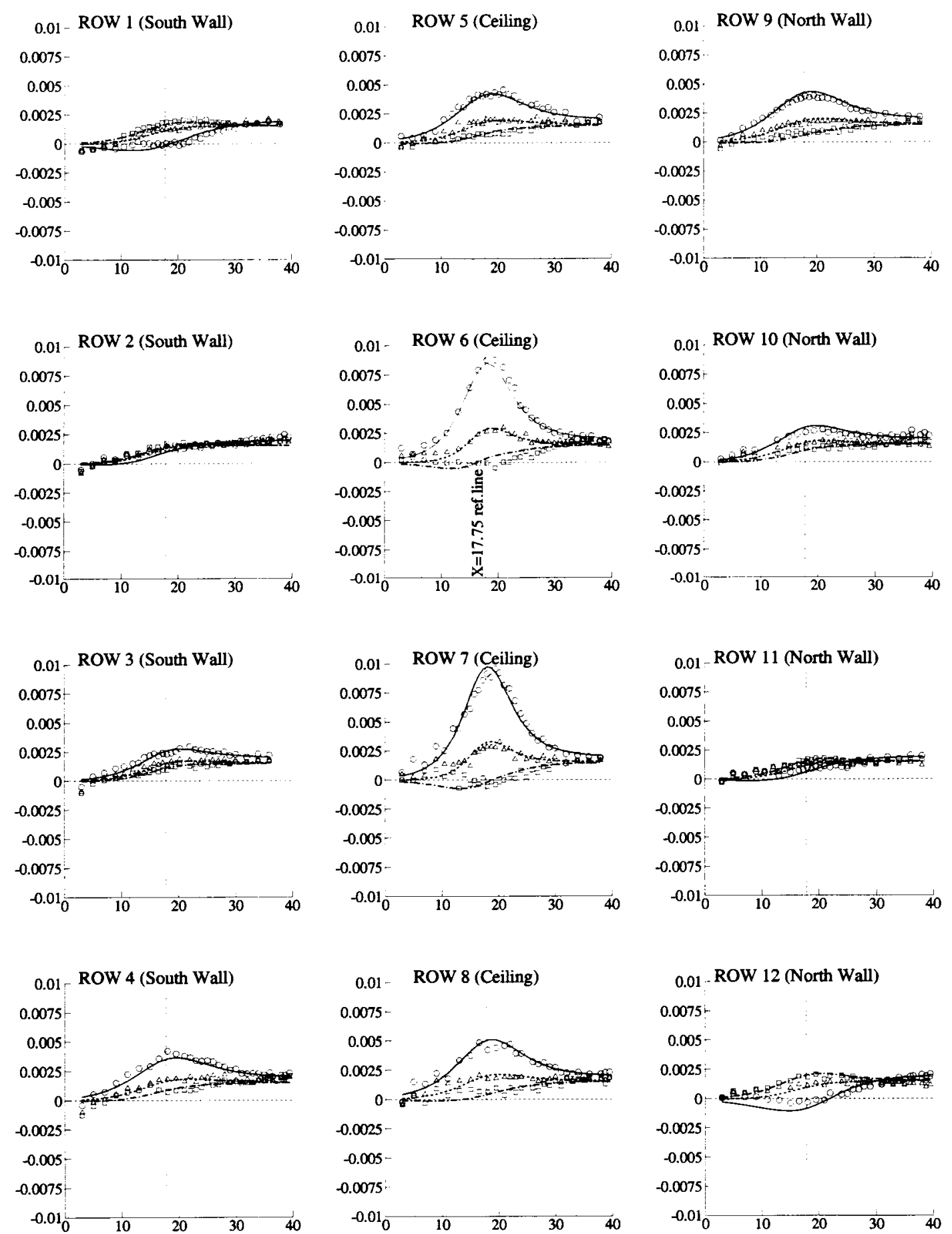

Figure 12. Measured and TWICS-fit wall signatures for the elliptical wing test. Symbols are incremental measurements; lines are TWICS-fit values. Three angles of attack $-3^{\circ}$ (squares, dash-dot line), $2^{\circ}$ (triangles, dashed line), $12^{\circ}$ (circles, solid line) are shown. $\mathrm{X}$-axis is the streamwise distance $\mathrm{X} ; \mathrm{Y}$-axis is the incremental normalized wall velocity. 

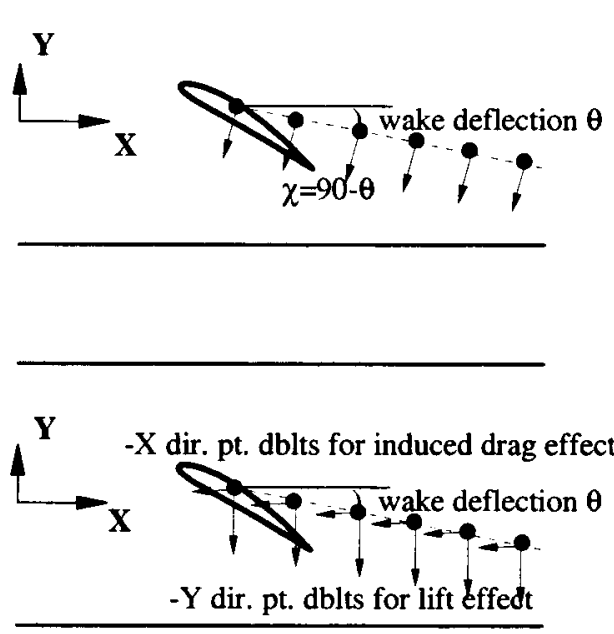

Figure 13. Part (a)( top), Heyson wake deflection model. Part (b) (bottom), Superposition used in the Heyson model.
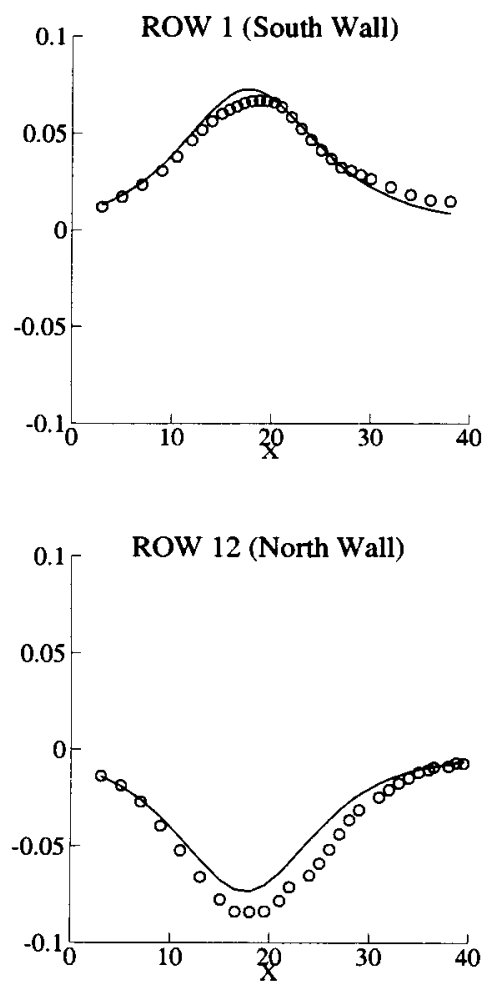

Figure 14. Comparison of measured incremental wall velocities (symbols) with computed values from a modified deflected wake TWICS code, $\alpha=32^{\circ}$.

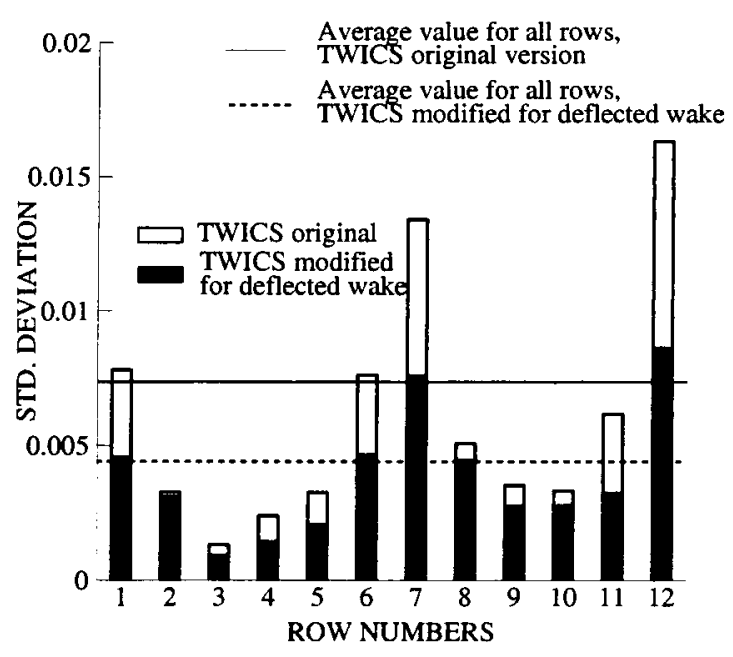

Figure 15. Improvement in the standard deviation of the TWICS fit to the measured wall pressures along each wall row with the deflected wake modification, example shown above is for $\alpha=32^{\circ}$.
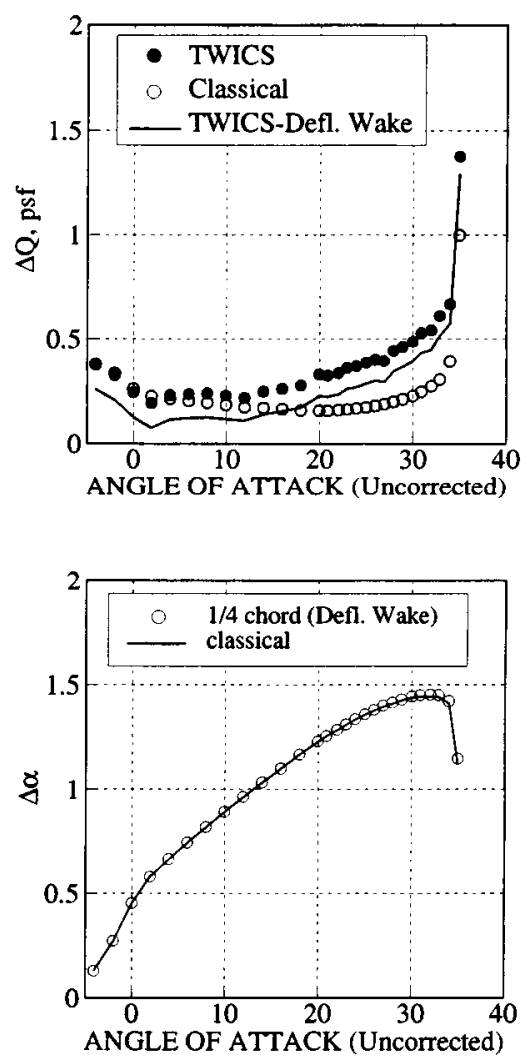

Figure 16. Improvement in wall corrections with the TWICS deflected wake modification. 